\title{
A System for Wireless Power Transfer of Micro-Systems In-Vivo Implantable in Freely Moving Animals
}

\author{
Enver G. Kilinc, Student Member, IEEE, Gilbert Conus, Chloe Weber, Basile Kawkabani, Senior Member, IEEE,
} Franco Maloberti, Fellow, IEEE, and Catherine Dehollain, Member, IEEE

\begin{abstract}
A system for wireless power transfer of microsystems in-vivo implantable in small animals is presented. The described solution uses a servo-controlled transmitter moved under the animal moving space. The solution minimizes the power irradiation while enabling animal speeds up to $30 \mathrm{~cm} / \mathrm{s}$. An $x-y$ movable magnetic coil transmits the required power with a level able to keep constant the received energy. A permanent magnet on board of the implantable micro-system and an array of magnetic sensors form a coil tracking system capable of an alignment accuracy as good as $1 \mathrm{~cm}$. The power is transferred over the optimized remote powering link at $13.56 \mathrm{MHz}$. The received ac signal is converted to dc voltage with a passive fullwave integrated rectifier and the voltage regulator supplies $1.8 \mathrm{~V}$ for the implantable sensor system. Experimental measurement on a complete prototype verifies the system performance.
\end{abstract}

Index Terms-Remote powering, wireless power transfer, implantable electronics, multi-sensor system, monitoring system, implantable micro-system, servo-controlled power transfer, $x-y$ rails, biomedical implant, system for freely moving animal, remotely powered sensor systems, in-vivo implantable system.

\section{INTRODUCTION}

$\mathbf{F}$ ABRICATION of electrochemical sensors directly on a silicon surface allows miniaturization of bio-sensor systems [1]. Especially, electrochemical sensors and electronic circuits are merged by growing carbon nanotubes on integrated circuit [2]. Therefore, this miniaturization step is essential to become widespread. These platforms help to monitor continuously different chemical and/or biological vital parameters as continuous monitoring is crucial for understanding the progression or regression trend of illness and applying correct treatments on the patient.

The rodents are commonly used animals for developing new treatments and research applications [3]-[7]. The size of these animals allow to stock larger number of animals in a certain

Manuscript received September 4, 2013; revised October 2, 2013; accepted October 2, 2013. Date of publication October 7, 2013; date of current version December 5, 2013. This work was supported by the Swiss National Funding through Sinergia Initiative. The associate editor coordinating the review of this paper and approving it for publication was Prof. Subhas C. Mukhopadhyay.

E. G. Kilinc, G. Conus, C. Weber, B. Kawkabani, and C. Dehollain are with the Institute of Electrical Engineering, Ecole Polytechnique Fédérale de Lausanne, Lausanne 1015, Switzerland (e-mail: enver.kilinc@epfl.ch; gilbert.conus@epfl.ch; chloe.weber@epfl.ch; basile.kawkabani@epfl.ch; franco.maloberti@unipv.it; catherine.dehollain@epfl.ch).

F. Maloberti is with the Institute of Electrical Engineering, Universitá degli Studi di Pavia, Pavia 27100, Italy (e-mail: franco.maloberti@unipv.it).

Color versions of one or more of the figures in this paper are available online at http://ieeexplore.ieee.org.

Digital Object Identifier 10.1109/JSEN.2013.2284815 space compared to other animals. In addition, the life span of the rodents is shorter hence they become adult quickly [8], [9]. Finally, the most essential feature of these animals is suitable for modification on their DNA sequence which permits to create DNA sequence similar to human genome [10]. However, since the size of mice are notably small, the implantable system has to be extremely small and light-weight. This forces the design of batteryless system to enable overall small size and weight.

Since the living condition has significant effects on credibility of measurement results, animals should be in a stressless and comfortable living area and move freely. Additionally, the animal must be in conscious in order to get reliable data from the measurements [11].

The major problems of remotely powered batteryless sensor systems are the size of the implantable platform and the continuity of the measurements without interruption. The overall size of the implantable device limits the size of the implant antenna or coil which influences the received power at the implantable device. Moreover, the distance between external antenna and implantable system changes the power transfer efficiency. Both issues determine the choice of the most efficient power transfer method. Our analysis concluded that the magnetically coupled inductive link is suitable for wireless power transfer accounting for the distance between the base station and the implantable devices and also the allowed device sizes [12], [13]. The magnetically coupled remote powering system is composed of an external and an internal units. The external unit is composed of power amplifier, supply controller, receiver and powering coil. The internal unit has implant coil, rectifier, voltage regulator, voltage level detector, transmitter and sensors.

The medium where the electrochemical sensors are placed need to be stabilized according to the applied reference voltage before the sensing is started [14]. Accordingly, this process needs to be powered without interruption. Otherwise, the medium is not biased properly and the measurement result will be wrong. In addition, the implantable system is placed inside a freely moving animal which increases the difficulties of continuous power transfer. The power transfer efficiency from the powering coil to the implant coil decreases when the implantable system moves from the center to the edges of the powering coil. Therefore, the received power of the implantable system is limited and non-uniform. In order to solve these issues, an Intelligent Remote Powering (IRPower) 


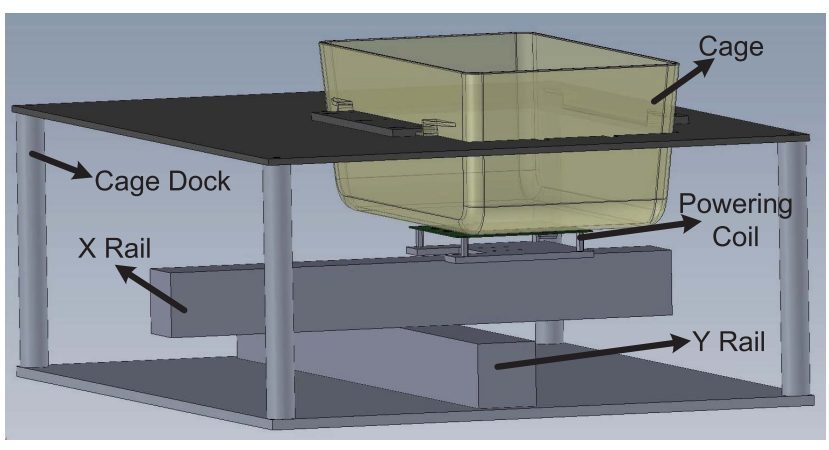

Fig. 1. 3-D model of IRPower system.

system is essential to track the freely moving animal and transfer the power continuously and uniformly [15]. Fig. 1 demonstrates the 3-D model of the IRPower system. The IRPower system can pursue an animal inside a living space and transmit the power wirelessly to the implantable unit thanks to the $\mathrm{X}$ and $\mathrm{Y}$ rails. The animal tracking is performed by the magnetic field sensors which follow the permanent magnet inside the animal.

This paper proposes the IRPower system which is based on tracking of freely moving animal and the power is transmitted dynamically to an implantable batteryless sensor system. Section II gives overview about the remotely powered implantable batteryless multi-sensor system. Section III presents the remote powering circuits and the optimized powering link. Section IV describes the IRPower system and how to track a moving animal in a living space. Section V presents a case study on IRPower system for freely moving rodents which includes the measured results. Section VI discusses about the important challenges of the IRPower system. Finally, Section VII concludes the paper.

\section{Remotely Powered Implantable Multi-Sensor SYSTEM}

The multiple sensor systems allow to sense different chemical compounds as well as vital signals on the same platform. Additionally, the use of multiple sensors on the same implantable system gives less invasion and optimizes the processing operations. Fig. 2 illustrates the conceptual design of the batteryless implantable multi-sensor system. The sensors are placed on the top of the 3-stage platform to create a good contact with the body. The electrochemical sensors detect the different types of compounds and chemical parameters. The targeted materials are mainly different drugs, and glucose. The temperature and $\mathrm{pH}$ of the implanted medium play a significant role on detection capability of the sensors. Therefore, the $\mathrm{pH}$ and temperature sensors are also placed on the top of the platform to obtain reliable measurement results by taking account of the effect of temperature and $\mathrm{pH}$ of the medium on sensors during the post-processing of the sensor data. The middle stage houses the electronic components, with integrated circuits used for power management, communication and sensor interface. Moreover, in the middle stage there is a storage capacitor, used to guarantee the longevity of the measurement. Finally, the implant coil is placed in the bottom of the platform in order to induce current from available magnetic field.

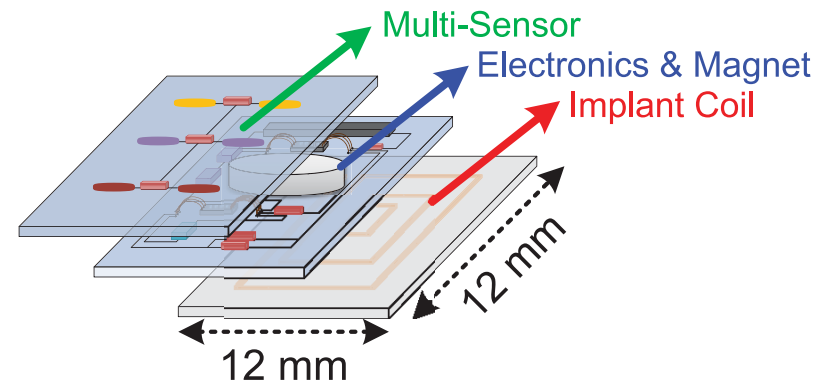

Fig. 2. Conceptual design of batteryless implantable multi-sensor system.

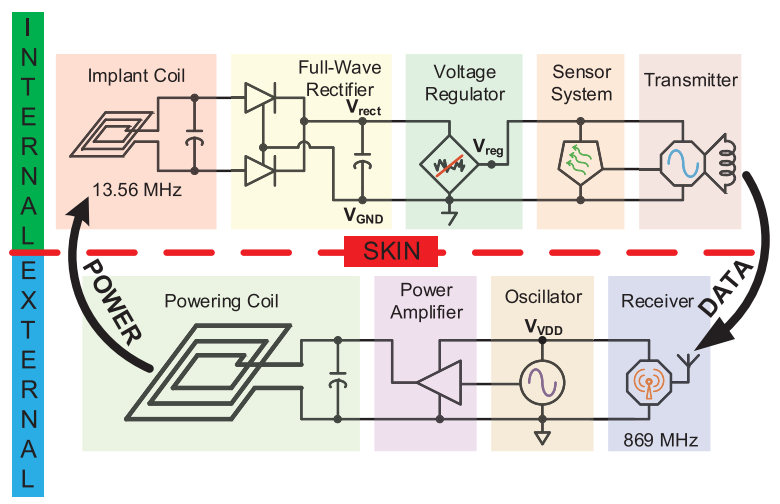

Fig. 3. Block diagram of implantable remotely-powered multi-sensor system.

The electronics of the implantable sensor system includes the pre-processing and data conversion needed by specific sensors and has in common service section for power management and data communication. Fig. 3 shows the block diagram of the standard sections of the implantable remotelypowered multi-sensor system. A power amplifier which drives a powering coil is followed by an optimized remote powering link [16]. The received AC signal is converted to DC voltage supply for the sensor system by efficient integrated circuits. The measured parameters in the animal body are transmitted by a low-power transmitter at $869 \mathrm{MHz}$ frequency to external unit [17].

\section{WiRELESS POWER TRANSFER}

The wireless power transfer distance can range from few $\mathrm{mm}$ up to $\mathrm{dm}$ for the system implanted in a freely moving animal [3], [6], [18]. Moreover, the magnetic field strength is inversely proportional to the cube of the distance $\left(1 / \mathrm{d}^{3}\right)$ between the powering and the implant coils [19]. Accordingly, a weakly coupled coils needs to be optimized to achieve maximum power transfer efficiency which is dominated by the limited efficiency of the remote powering link. The remote powering link is optimized for $30 \mathrm{~mm}$ wireless power transfer distance by the equations are given in [16]. The power transfer efficiency is proportional to the coupling coefficient square and the quality factors of the coils $\left(\mathrm{k}^{2} \mathrm{Q}_{\text {pow }} \mathrm{Q}_{\text {imp }}\right)$. Therefore, the main goal of the optimization is to maximize these terms. The coils have several geometric parameters such as outer diameter, inner diameter, spacing between the conductors, the width and height of the conductors, number of turns, etc., 


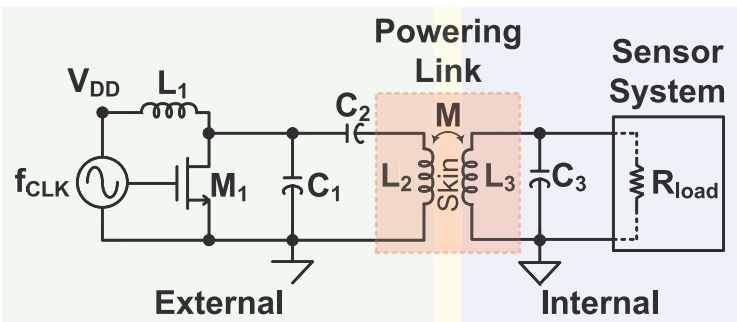

Fig. 4. Wireless power transfer over remote powering link.

which correspond to different coupling coefficient and quality factors value in the link. The proposed optimization method in [16] finds the optimal coil geometries and verifies the remote powering link by a 3-D Finite Element Model (FEM) simulator. The optimal size for the powering and the implant coils are found as $80 \times 80$ and $12 \times 12 \mathrm{~mm}^{2}$, respectively.

Fig. 4 shows the power amplifier and the remote powering link. The powering coil is driven by a class-E power amplifier due to its high drain efficiency. The power is transferred over the optimized remote powering link at $13.56 \mathrm{MHz}$ which is a specialized band for inductive applications. The induced AC voltage on the implant coil is converted to DC voltage by a passive full-wave rectifier. The forward voltage drop across a diode-connected transistor is high which decreases the power efficiency of the rectifier. In order to decrease the voltage drop, the high-threshold transistor can be replaced by a low or zero threshold transistor. However, the leakage current is increased due to the non-disabled transistor. Therefore, the rectifier, in this paper, uses the charge-storage technique which decreases the forward voltage drop on the diode connected high-threshold transistor [17]. This technique reduces the gatesource voltage $\left(V_{G S}\right)$ of the transistor and allows to achieve the power efficiency of $80 \%$ with a passive rectifier. Furthermore, the ripples at the rectifier output are suppressed by a high-speed voltage regulator. A single-stage OTA is used to suppress the ripples at the remote powering frequency and a clean $1.8 \mathrm{~V}$ supply voltage is created.

\section{IRPOWER SYSTEM}

In a previous study, the optimum outer dimension of the powering coil was found to be $80 \mathrm{~mm}$ [16]. A possible solution which enables us to cover the base of the living space, is to use an array of coils are placed under the living space as shown in Fig. 5. A tracking system allows delivering the optimum power to the implant by turning on the most appropriate powering coil [20]. A permanent magnet is placed in the middle of the implant coil; the magnetic field sensors detect the DC magnetic field of the magnet and give a voltage response to corresponding magnetic strength. Consequently, the analog output voltages of the sensors monitor the position of the animal. Accordingly, the appropriate coils are turned on and irrelevant coils are turned off. Additionally, the power amplifier supply can be adjusted for optimum power transfer. However, there are still dead zones where the power cannot be effectively transferred to the implantable system. Moreover, the power transfer efficiency fluctuates and it is not always in the most efficient point. In addition, some studies present that

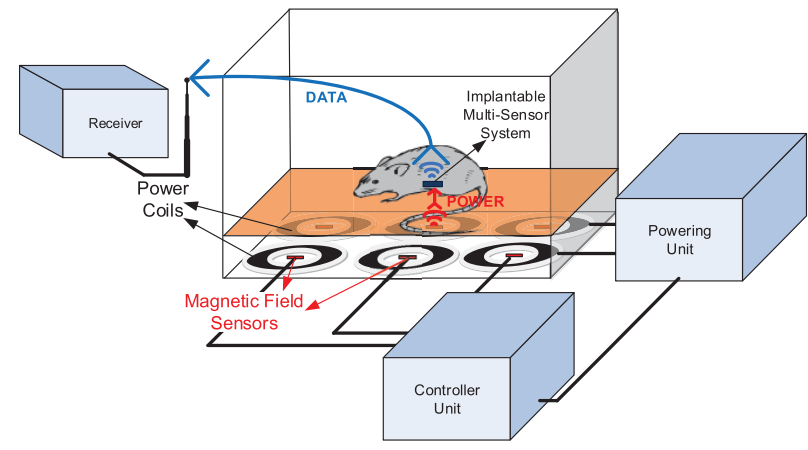

Fig. 5. Scenario for magnetically coupled remote powering system for freely moving animal.

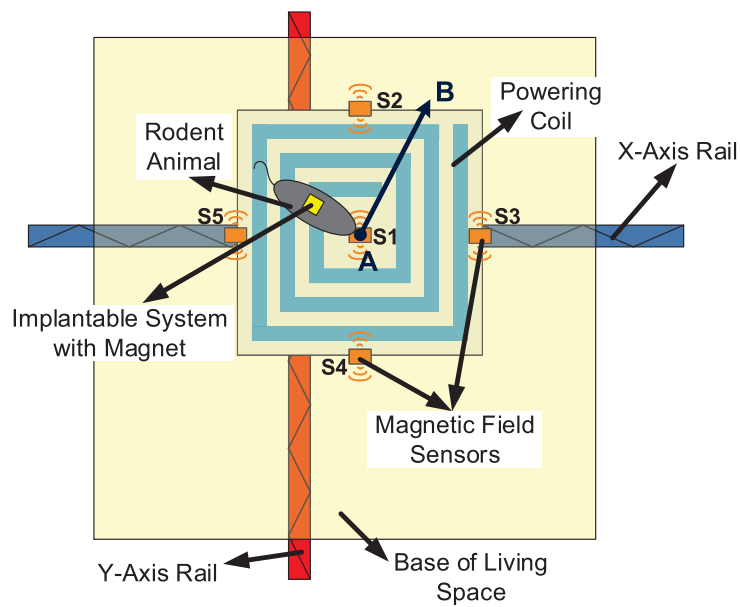

Fig. 6. IRPower system for freely moving animal.

the multi-coil remote powering links reduce the effect of coil misalignment effect and achieve good power transfer efficiency [21]. However, these multi-coil structures compensate the efficiency drop until a certain misalignment point. After this point, the power transfer efficiency decreases drastically. In remotely powered system for freely moving animal, the wireless power transfer should be continued for any position of animal without disturbing the animal. If the animal stays in the edge of the powering coil, the power transfer efficiency decreases to less than $1 \%$ and the transferred power level needs to be increased to continue the remote powering. The high power also increases the temperature of the living space and disturbs the animal. Accordingly, the power transfer efficiency should be kept always maximum level not to disturb the animal.

In order to fix aforementioned limits, the IRPower system is proposed. Fig. 6 shows the IRPower system for freely moving animal. Two rails ( $\mathrm{X}$ and $\mathrm{Y}$ rails) are installed under the cage to move the powering coil. In addition, the cage is stabilized with a cage dock as illustrated in Fig. 1. The system defines the relative position of the implantable multi-sensor system with a permanent magnet. A magnetic field sensor placed on the powering coil detects the DC component of the magnetic field. The IRPower system follows the DC magnetic field strength and moves the powering coil under the base of the living space to track the position of the freely moving animal. Therefore, power is always optimally delivered with high efficiency to the implantable system. 


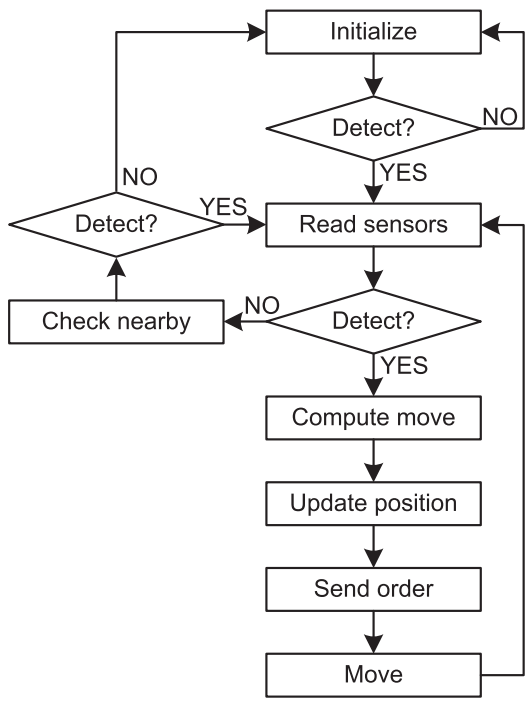

Fig. 7. Simplified flowchart of IRPower algorithm.

Fig. 6 explains the operation of the IRPower system. The main goal of the system is to keep the animal in the center of the powering coil. Accordingly, several magnetic field sensors are placed around the powering coil. Suppose that the animal moves from point $A$ to point $B$. When the animal is at the point $A$, the sensor $S 1$ which is located in the center of the powering coil has the maximum output voltage compared to the other sensors on the edges of the powering coil. When the animal moves from point $A$ to point $B$, the output voltage of the sensor $S 1$ will decrease and the sensors $S 2$ and $S 3$ will increase. Accordingly, the IRPower system moves the powering coil to north-east direction until the output of the sensor $S 1$ is again maximized compared to other sensors.

As an additional information, IRPower system monitors the movements of the animal which can be recorded and used to analyze the status and attitude of the animal. The data shows how much the animal runs, walks, pauses, and where the animal stays mostly in the living space. In addition, it can be used to estimate the energy consumption of the animal.

Fig. 7 shows the simplified flowchart of the IRPower algorithm. The IRPower system starts with initialization of the system. The initialization phase resets all memories which store the tracking data and puts the powering coil to the origin point which is reference point, defined by the user, for tracking the animal. The detection phase follows the initialization phase. The IRPower system starts to search for the animal in the cage. The $\mathrm{X}$ and $\mathrm{Y}$ rails help the magnetic field sensors sweep all the base of the living space until the sensors detect the magnet implanted inside the animal. The $\mathrm{X}$ and $\mathrm{Y}$ rails move the powering coil in the position that maximizes the output of sensor $S 1$ after the magnet is detected. The wireless power transfer is turned on after the powering coil is cocentered with the implant coil. If the sensors cannot detect the magnet after sweeping all the base of the living space, the system returns to initialization step. The IRPower system starts tracking the magnetic field sensors output for any movement of animal. When the animal moves, the outputs of the sensors change accordingly. This allows the system to compute the

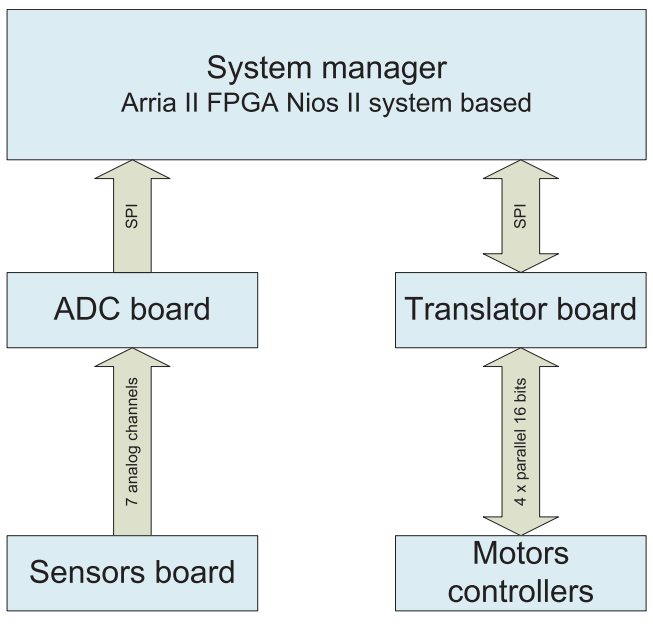

Fig. 8. Block diagram of IRPower system.

necessary movement of the powering coil, updated after each movement is computed.

Calculation of the animal position by a microcontroller is difficult since many complex mathematical equations should be calculated such as integrals. In order to reduce the computation time, a Look-Up Table (LUT) is created by averaging 20 samples taken from each sensor for a certain vertical distance between the animal and the sensors. Each magnet position on the powering coil has a corresponding sensors outputs value as shown in Fig. 12 which is stored in the LUT. Therefore, the position of the animal is computed by comparing the sensors outputs with the LUT. In addition, the movement and the next position are stored on memories for tracking of the animal. Obviously, the move order sent to motor controller activates the $\mathrm{X}$ and $\mathrm{Y}$ rails. The IRPower system returns "Read sensor" step to sense the new movement.

The rodent animal can stand up on its hind legs and explore the area. This action takes around $5 \mathrm{~s}$ according to the several videos which are recorded in laboratory environment. The IRPower system can understand that the animal stands up as the value of the sensor $S 1$ will decrease and none of the other sensors will change their outputs. If this case is detected during "Read sensor" phase, the system awaits for $5 \mathrm{~s}$ to guarantee the animal returned from its stand up position to normal position. If the delays of $5 \mathrm{~s}$ elapsed and the value of sensor $S 1$ does not change back to its maximum level, it means the animal stands down but not the same place where it stood up. Accordingly, the IRPower system starts to relocate the mouse by checking nearby the latest known position. In case the IRPower system cannot find the magnet in the nearby region, the IRPower system returns to the initialization phase.

The IRPower system consists of 5 main blocks. Fig. 8 represents the block diagram of the IRPower system. An FPGA (Arria II GX) [22] realizes the controller. A PCB houses both the linear output magnetic field sensors (AD22151) and the powering coil, as Fig. 9 shows [23]. The output of the sensors is an analog voltage ranging between 0 and $5 \mathrm{~V}$ depending on the magnetic field strength and the pole of the magnet. The sensors drive an analog to digital converter (ADC) board through a cable. The ADC (ADC128S102) is a 


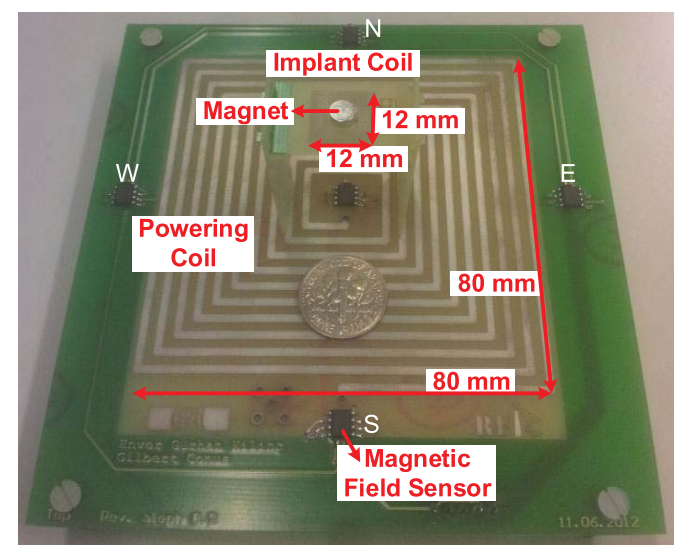

Fig. 9. Optimized remote powering link with magnetic field sensors.

low-power, 8-channels, and 12-bits converter with sample rate up to $1 \mathrm{MS} / \mathrm{s}$ [24]. A serial peripheral interface (SPI) bus links the ADC and the FPGA.

Two motor controller drive the $\mathrm{X}$ and $\mathrm{Y}$ rails (RS106-C1-N) [25]. The motor controllers are connected to the FPGA. The FPGA runs with $2.7 \mathrm{~V}$ logic voltage, it is also compatible with $3.3 \mathrm{~V}$. The FPGA communicates with motor controller over SPI bus. However, two motor controllers operate with $24 \mathrm{~V}$ logic voltage and have 16 bits parallel input and output. A voltage level translator board ensures compatibility between the FPGA and the motor controllers. The translator board is composed of 16 bits I/O expanders (MCP23S18) and high-voltage optocouplers (FODM8801C) [26], [27]. The I/O expanders have bidirectional input-output pins which handle the serial to parallel and parallel to serial data conversions between the FPGA and the motor controllers. In addition, the optocouplers provide an isolation between the I/O expanders and motor controllers and a translation between the different voltage levels.

The IRPower system can sweep all the bottom of the cage (with size of $18 \times 34 \mathrm{~cm}$ ) in less than 75 seconds. The system obtains 20 samples from each sensor and computes the average values of these samples in order to reduce the effect of noise. The acquired values are transmitted to the FPGA in 16-bit packets. Therefore, the "Read sensor" step takes $308 \mu$ s to transmit 2240 bits ( 7 sensors, 20 samples, 16-bit packet) and compute the average values. Although the FPGA has a system clock rate of $150 \mathrm{MHz}$, the ADCs are limited with $10 \mathrm{MHz}$ clock rate. Therefore, the duration of the reading phase is dominated by the clock of the ADCs.

The IRPower system determines the next movement and records the new position on the memories in $1.5 \mu \mathrm{s}$. The command is transmitted to rails in $5 \mathrm{~ms}$ due to requirement of the motor controllers. The movement of the rails can be adapted due to the animal movement. The speed and acceleration/deceleration of the rails and the distance to move can be adjusted.

\section{CASE STUdy: IRPOWER SYSTEM FOR FREely MOVING RODENTS}

The rodents are very active animals. For example, the mice can easily reach speed of $4 \mathrm{~m} / \mathrm{min}$ [10]. This defines the

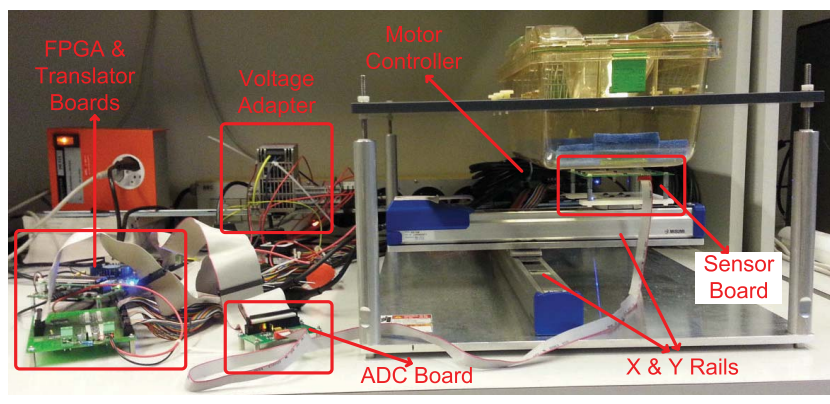

Fig. 10. Test setup for proposed wireless power transfer and animal tracking system.

speed of rails in our system, rails chosen with maximum of $18 \mathrm{~m} / \mathrm{min}$, much faster than the animal. The total length of $\mathrm{X}$ and $\mathrm{Y}$ rails are 200 and $350 \mathrm{~mm}$ respectively as shown in Fig. 1. In addition, the rails have 255 positioning points. Therefore, the minimum step size of the $\mathrm{X}$ and $\mathrm{Y}$ rails are $0.78 \mathrm{~mm}$ and $1.37 \mathrm{~mm}$ respectively.

Fig. 9 shows the optimized remote powering link with the magnetic field sensors. The permanent magnet is placed in the middle of the implant coil. A neodymium $(\mathrm{NdFeB})$ type of magnet maximizes the DC magnetic field which has the N52 magnetization grade [28]. The magnetic flux density $\left(B_{R}\right)$ value of the magnet is $1.42-1.47 \mathrm{~T}$ and the energy product $(\mathrm{B} \times \mathrm{H})$ is $380-422$ MGOe. The diameter of the magnet is $5 \mathrm{~mm}$ with $2 \mathrm{~mm}$ height.

The test setup shown in Fig. 10 validates the operation and analyze the performance of the system. The FPGA, translator, ADC boards and motor controllers are shown in Fig. 10. Moreover, the implementation of $\mathrm{X}$ and $\mathrm{Y}$ rails with sensor board is outlined. The implant coil is placed $30 \mathrm{~mm}$ away (vertically) from the powering coil to simulate the cage environment where the distance between the powering and the implant coils is increased due to the housing condition of animal.

Fig. 11 shows the sensor board and 7 magnetic field sensors. The number of the sensors is 7 (6 sensors on the edges and 1 sensor in the center as shown in Fig. 11). Compared to Fig. 9 the arrangement increases the overall sensitivity of the sensor board and the shared sensible area of the sensors. The need of a small implanted magnet gives rise to a range of sensitivity which is maximum under the sensors and goes to zero in some region of the in the 2-D area. The sensor board is divided into small sensing regions as shown in Fig. 11 to create the sensitivity map of the entire board. Each red square $(5 \times 5 \mathrm{~mm})$ shows one sensing region. Accordingly, the magnet is placed on each of these regions and the output channels of the ADC are measured by the FPGA for each sensing region. The ADC is chosen as 12-bit resolution since the magnetic field sensors have $0.4 \mathrm{mV} / \mathrm{G}$ internal sensitivity. The output of the ADC changes between 0 and 4095 digital levels which corresponds to 0 and $5 \mathrm{~V}$ of the magnetic field sensor output, respectively. In order to compare easily, the outputs of the sensors are also presented in digital coded levels. Fig. 12 shows the 2-D sensitivity map of the entire sensor board for 


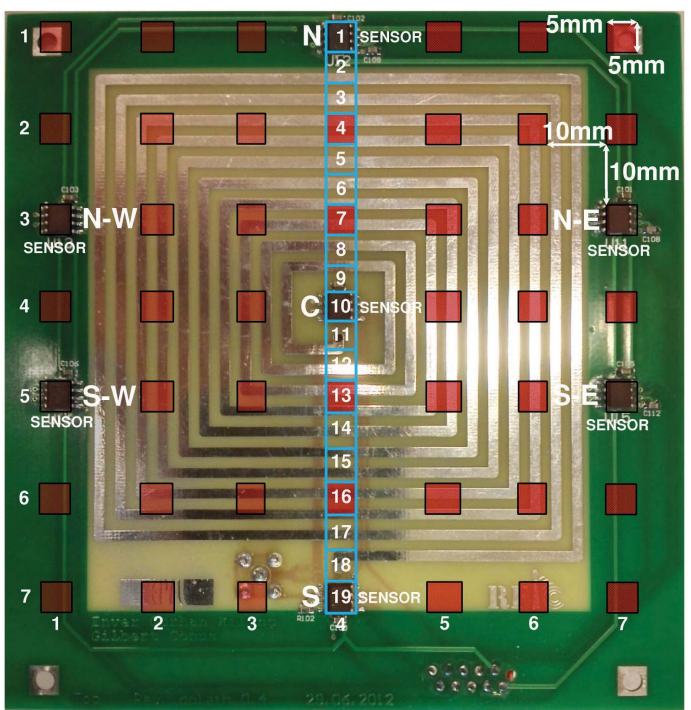

Fig. 11. Sensor board and 7 magnetic field sensors.

$\square$ North-East $\square$ South-East $\square$ South-West $\square$ North-West $\square$ North $\square$ South $\square$ Center

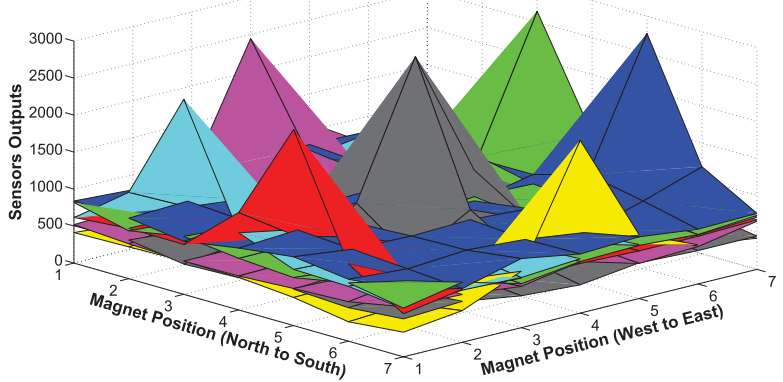

Fig. 12. 2-D sensitivity map of sensor board for different magnet position.

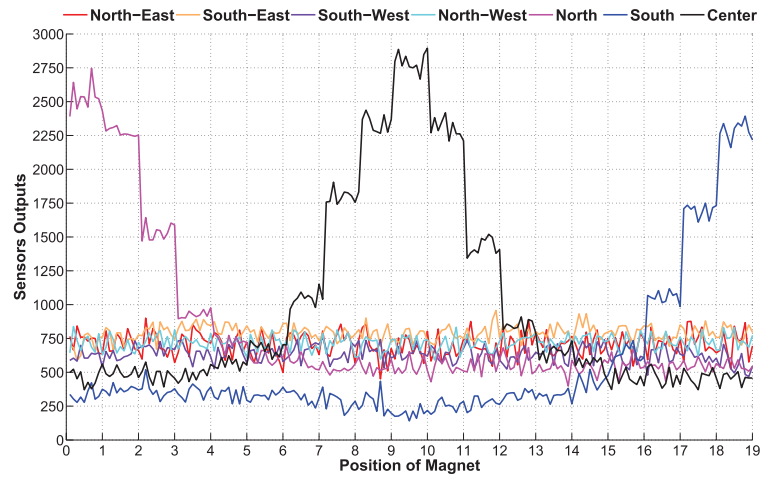

Fig. 13. Magnetic field sensors 1-D response while magnet moving from north to south.

different magnet position. Additionally, the magnet is swept from north sensor to south sensor as illustrated in blue grid in Fig. 11. Fig. 13 shows the sensors output in detail due to position of the magnet. The motion of the magnet is detected by following the sensors outputs from north to south as in Fig. 13.

The power is transferred over the optimized remote powering link as shown in Fig. 9. The received $\mathrm{AC}$ signal is

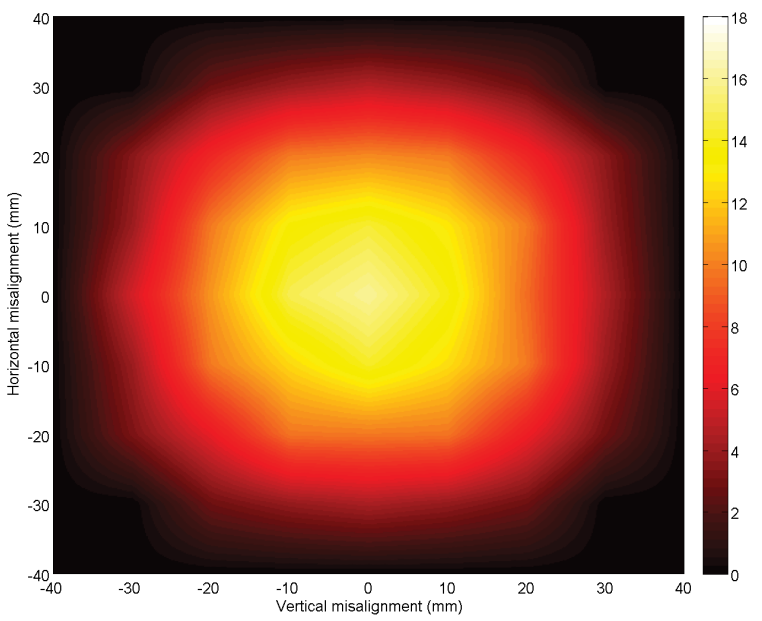

Fig. 14. Power transfer efficiency distribution on powering coil including power amplifier, remote powering link, and rectifier efficiencies.

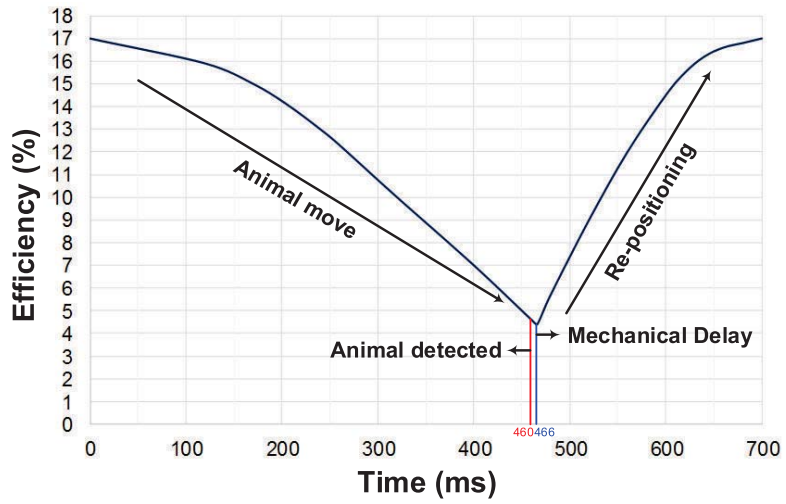

Fig. 15. Worst case scenario for power transfer efficiency decrease due to IRPower system delay.

converted with DC supply voltage by the full-wave rectifier. The power efficiency of the rectifier is measured as $77.5 \%$ for $1.7 \mathrm{~mW}$ load power. The power supply rejection of the voltage regulator is measured as $60 \mathrm{~dB}$ for the line regulation and at the remote powering frequency. The implantable circuits for remote powering are fabricated in 0.18 CMOS process. Fig. 14 shows the power transfer efficiency distribution on the powering coil including the power amplifier, the remote powering link, and the rectifier efficiencies. The efficiency decreases from $17 \%$ to less than $1 \%$ for $1.7 \mathrm{~mW}$ of load power when the implantable system moves from the center of the coil to the edges, respectively.

Assuming that the animal moves at speed of $7 \mathrm{~cm} / \mathrm{s}$ and the rails move at speed of $30 \mathrm{~cm} / \mathrm{s}$ with $400 \mathrm{~cm} / \mathrm{s}^{2}$ acceleration. Fig. 15 shows the power transfer efficiency change due to the animal move and the system delay. In the worst case scenario, even if IRPower system detects the animal close to edge where the efficiency drops to $4.7 \%$, there is only $5.4 \mathrm{~ms}$ mechanical delay after the animal is detected which corresponds to decrease the efficiency less than $0.5 \%$ and the system is repositioned in less than $250 \mathrm{~ms}$. However, the system delay can be reduced and IRPower system can 


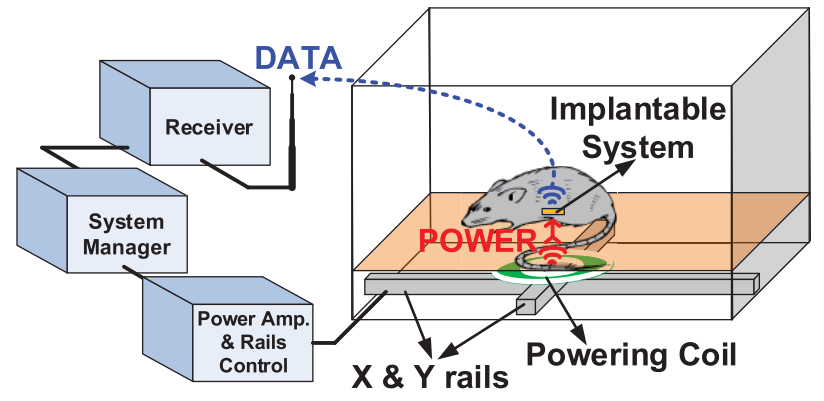

Fig. 16. Wireless power transfer with power feedback loop.

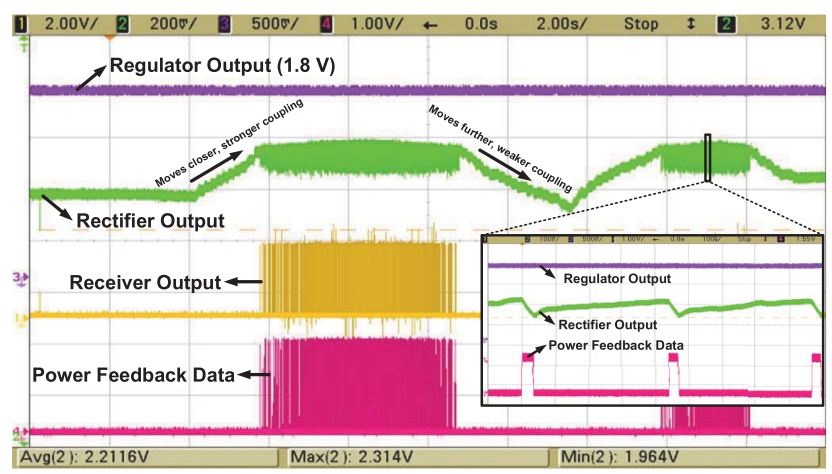

Fig. 17. Remote powering signals (rectifier, regulator voltages) and power feedback data.

be improved by increasing the size of the implanted magnet which makes the animal more detectable. Also, increasing the speed of the rails helps to reduce the re-positioning delay. Therefore, the power transfer efficiency is kept at always maximum level.

Many studies show that the rodents spend most of the time in a horizontal position (on their four feet) [10], [29]. Accordingly, assuming that the implant coil moves parallel to the powering coil, the power transfer efficiency changes during the movement, hence the received power level by the implantable system also changes. In order to guarantee the continuous operation and deliver the sufficient power to the implantable system, the power feedback loop is proposed. Fig. 16 shows the wireless power transfer with power feedback loop. The power feedback loop adjusts the transferred power level according to demand of the implantable system. The power level of implant is monitored and the power feedback data is transmitted to the external receiver. The system manager controls the transferred power level by changing the supply of the power amplifier. Fig. 17 shows the remote powering signals (rectifier, regulator voltages) and the power feedback data. The received AC signal is rectified and the rectifier output is compared with a reference voltage. The comparison result, power feedback data, is transmitted to the external receiver and the supply voltage of the power amplifier is adjusted according to this data. If the power level in the implantable system is insufficient, the power amplifier increases the output power. On the contrary, if the power is more than necessary level, the power amplifier reduces the output power not to damage the implant. In order not to damage the PA circuit, the output

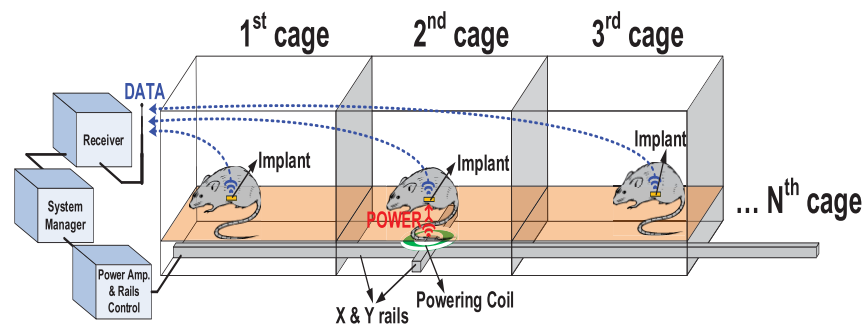

Fig. 18. Scenario for multi-cage monitoring.

power level is limited. Therefore, the rectifier output drops under $2.2 \mathrm{~V}$ when the implant coil moves further but the regulator output is not perturbed.

The animal can stand up and the implant coil rotates and changes its orientation. This angular misalignment reduces the coupling between the coils and the power transfer efficiency. The decrease can be compensated by also rotating the powering coil to be same orientation with the implant coil. However, the animal moves on four feet most of the time. Therefore, the powering coil is placed close to the bottom of the living space of the animal to minimize the vertical distance and increase the coupling between the coils. Accordingly, it is impossible to rotate the powering coil to change the magnetic field orientation. Unless the implantable system is perpendicular to the powering coil where the magnetic field strength is zero and the power transfer is almost impossible, the power feedback loop keeps the rectifier voltage at sufficient level to create $1.8 \mathrm{~V}$ supply voltage without perturbation.

The electrochemical sensors which will be powered by IRPower system needs $200 \mathrm{~s}$ to complete a cyclic-voltammetry measurement for detecting the compound [30]. Although IRPower system relocates the implantable system in less than $75 \mathrm{~s}$, if the wireless power transfer is perturbed, a $200 \mathrm{mF}$, $3.3 \mathrm{~V}$ storage capacitor [31] can guarantee $200 \mathrm{~s}$ duration to complete the measurement for $1 \mathrm{~mA}$ load current.

$$
\Delta t=\frac{\Delta V \cdot C}{i_{\text {load }}}=\frac{(3.3-2.2) \cdot 0.2}{0.001}=220 \mathrm{~s}
$$

The storage capacitor would also increase the overall size and weight of the implant. On the other hand, using a storage capacitor allows to achieve stand-alone implants and multicage monitoring. Fig. 18 illustrates a scenario for multi-cage monitoring. Each animal is detected in the cage and the implant is charged by IRPower system [15]. When the implant is charged, the implant transmits information about the charge is completed and IRPower moves next cage to detect animal and charge the implant. IRPower returns first cage when all cage is finished. The data transmitted from the implants are received by the multi-channel receiver. The number of the cage can be increased according to the storage capacitor size and the charging time of the each implant.

Fig. 19 presents the proof of concept of the IRPower system for a freely moving animal. In order to simulate freely moving animal, a freely moving robot is used and a permanent magnet is attached to the bottom of the robot. The IRPower system can track the freely moving robot easily and the $\mathrm{X}$ and $\mathrm{Y}$ rails are adapted according to the movement of the robot. 


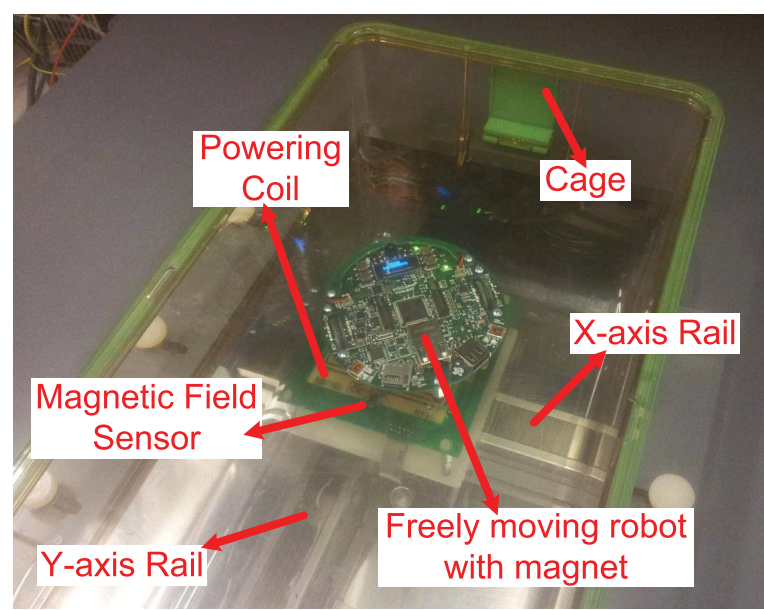

Fig. 19. Proof of concept of IRPower system for freely moving animal.

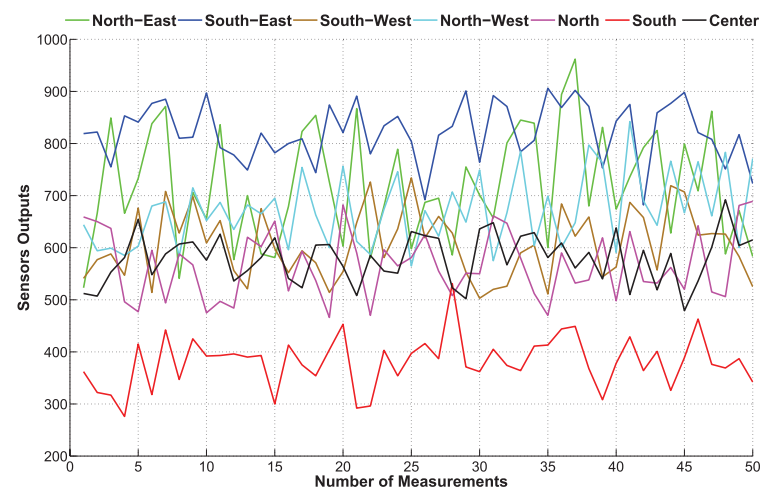

Fig. 20. Magnetic field sensors response without magnet.

The background sound levels in the rodent housing rooms are around $80 \mathrm{~dB}$ [32]. The sound pressure level of the rails is $70 \mathrm{~dB}$ when the rails move at maximum speed [25] which is comparable with other electrical devices in the housing rooms [33].

\section{DISCUSSION}

The performance of IRPower system depends on the offset and noise of the magnetic field sensors. Fig. 20 shows the magnetic field sensors responses without magnet, not placed on the sensor board. The sensors have different noise levels. Indeed, the outputs of the north-east sensor and the middle sensor change from 523 to 962 and from 479 to 692 , respectively. More important than noise is the offset values of the sensors. All the sensors have different offset values. In the specific case, the south sensor and the south-east sensor have the lowest offset and the highest offset values among the sensors, respectively. Therefore, the threshold values to sense the presence of the magnet and the maximum output value of the sensors when the magnet is above the sensor change from sensor to sensor as shown in Fig. 13. Additionally, the threshold values should be defined individually for all the sensors. Accordingly, IRPower system needs to be calibrated during the initialization phase due to the different offset values

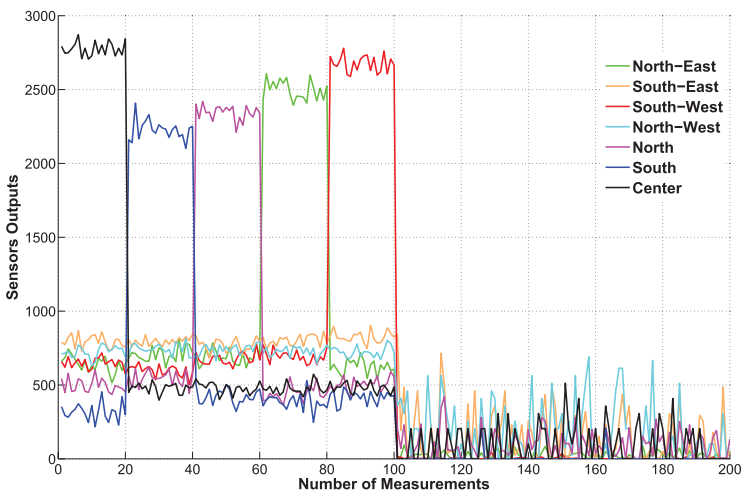

Fig. 21. Remote powering effect on magnetic field sensors.

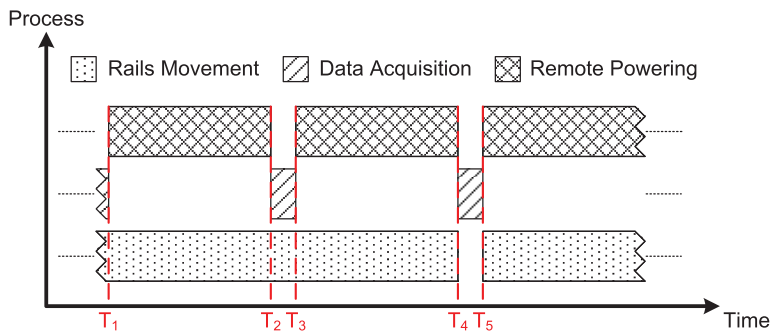

Fig. 22. Real-time parallel processing time schedule.

and also the changing vertical distance between the magnet and sensors.

In some applications, the implantable system demands more power. Therefore, the transferred power needs to be increased. Although the magnetic field sensors have low frequency response (cut-off frequency of $5.7 \mathrm{kHz}$ ), the magnetic field sensors saturate after a certain transferred power level $(200 \mathrm{~mW})$ and do not detect the implanted magnet. Fig. 21 shows the remote powering effect on the magnetic fields sensors. The magnetic field sensors are able to detect the presence of the magnet properly when the powering coil does not generate any magnetic field. When the remote powering is turned on and the powering coil starts to generate larger magnetic field than the implanted magnet (after 100th measurement in Fig. 21), all the sensors are affected from magnetic field generated by the powering coil and the outputs of the sensors are saturated. Therefore, the sensors does not follow the magnet any more. In such case, one of the solutions is to increase the magnet size which generates higher magnetic field. However, this solution is inconvenient for implantable systems for small animals. Second solution is to perform the tracking and the remote powering asynchronously.

The remote powering affects the tracking system drastically. Therefore, the tracking system and the remote powering system do not work simultaneously. The remote powering must stop while the tracking system acquires data from the magnetic field sensors. Therefore, all the processes should be performed in parallel. Fig. 22 shows the real-time parallel processing time schedule. The remote powering and the data acquisition processes are not performed simultaneously. When the remote powering is stopped for the data acquisition process (around $300 \mu \mathrm{s}$ ), the implantable system can still continue 
to its operation by using the energy stored on the storage capacitor. The previous study presented in [34] shows that if a signal frequency is more than $10 \mathrm{~Hz}$, this signal is able to track the animal movement. Accordingly, the frequency of the data acquisition is not critical. The data acquisition can also be applied while the rails move. In opposite to sequential processing in Fig. 7, the data acquisition does not need to wait to end of the movement of the rails. Therefore, the data from the sensors is acquired more frequently (around $400 \mathrm{~Hz}$ ) which helps to track the freely moving animal more easily.

\section{CONCLUSION}

This paper presents a system for wireless power transfer of micro-systems in-vivo implantable in freely moving animals. The batteryless implantable micro-system is able to monitor the organic compounds, $\mathrm{pH}$ and temperature continuously for long-term duration. Therefore, the batteryless implantable micro-system needs to be powered continuously. This paper proposes a servo-controlled transmitter moved under the animal moving space. The $\mathrm{X}-\mathrm{Y}$ rails are used to place the powering coil to achieve maximum coupling with the microsystem. A permanent magnet on board of the implantable micro-system and an array of magnetic sensors form a coil tracking system capable of an alignment accuracy as good as $1 \mathrm{~cm}$. The powering coil is controlled by a suitable algorithm which locates the animal in the living space and adapts the rails according to the animal movement. Additionally, the tracking system is also capable of recording all the movement of the animal in order to analyze the animal behavior. The complete prototype confirms that the tracking system, enables the animal speeding up to $30 \mathrm{~cm} / \mathrm{s}$, is fast enough to track the freely moving animal easily.

\section{ACKNOWLEDGMENT}

The authors thank to F. Mazzilli, G. Yilmaz, M. Azizighanad, and O. Atasoy for their valuable feedback and help. Also, the authors are grateful to X. Warot and R. Doenlen from the Life Science Department, EPFL, for their supports on rodent animals.

\section{REFERENCES}

[1] M. M. Ahmadi and G. Julien, "A wireless-implantable microsystem for continuous blood glucose monitoring," IEEE Trans. Biomed. Circuits Syst., vol. 3, no. 3, pp. 169-179, Jun. 2009.

[2] S. Carrara, A. Cavallini, V. Erokhin, and G. De Micheli "Multi-panel drugs detection in human serum for personalized therapy," Biosens. Bioelectron., vol. 26, no. 9, pp. 3914-3919, 2011.

[3] P. Cong, W. H. Ko, and D. J. Young, "Wireless batteryless implantable blood pressure monitoring microsystem for small laboratory animals," IEEE Sens. J., vol. 10, no. 2, pp. 243-254, Feb. 2010.

[4] D. Fan, D. Rich, T. Holtzman, P. Ruther, J. W. Dalley, A. Lopez, et al., "A wireless multi-channel recording system for freely behaving mice and rats," PLoS One, vol. 6, no. 7, pp. 22033-1-22033-3, 2011.

[5] T. K. Givrad, J.-M. I. Maarek, W. H. Moore, and D. P. Holschneider, "Powering an implantable minipump with a multi-layered printed circuit coil for drug infusion applications in rodents," Ann. Biomed. Eng., vol. 38, no. 3, pp. 707-713, 2010.

[6] C. T. Wentz, J. G. Bernstein, P. Monahan, A. Guerra, A. Rodriguez, and E. Boyden, "A wirelessly powered and controlled device for optical neural control of freely-behaving animals," J. Neural Eng., vol. 8, no. 4, pp. 046021-1-046021-10, Jun. 2011.
[7] D. Russell, D. McCormick, A. Taberner, P. Nielsen, P. Hu, D. Budgett, et al., "Wireless power delivery system for mouse telemeter," in Proc. IEEE BioCAS, Nov. 2009, pp. 273-276.

[8] V. Reinhardt and A. Reinhardt, Comfortable Quarters for Laboratory Animals, 9th ed. Washington, DC, USA: Animal Welfare Inst., 2002.

[9] S. A. G. Willis-Owen and J. Flint, "The genetic basis of emotional behaviour in mice," Eur. J. Human Genet., vol. 14, no. 6, pp. 721-728, 2006.

[10] J. N. Crawley, What's Wrong With My Mouse: Behavioral Phenotyping of Transgenic and Knockout Mice, 2nd ed. New York, NY, USA: Wiley, 2007.

[11] Q. Wang, H. R. Brunner, and M. Burnier, "Determination of cardiac contractility in awake unsedated mice with a fluid-filled catheter," Amer J. Physiol. Heart Circle Physiol., vol. 286, no. 2, pp. 806-814, 2004.

[12] D. C. Yates, A. S. Holmes, and A. J. Burdett, "Optimal transmission frequency for ultralow-power short-range radio links," IEEE Trans. Circuits Syst. I, Regular Papers, vol. 51, no. 7, pp. 1405-1413, Jul. 2004.

[13] M. Sawan, Y. Hu, and J. Coulombe, "Wireless smart implants dedicated to multichannel monitoring and microstimulation," IEEE Circuits Syst. Mag., vol. 5, no. 1, pp. 21-39, Mar. 2005.

[14] A. Cavallini, C. Baj-Rossi, S. Ghoreishizadeh, G. De Mi, and S. Carrara, "Design, fabrication, and test of a sensor array for perspective biosensing in chronic pathologies," in Proc. IEEE BioCAS, Nov. 2012, pp. 124-127.

[15] E. G. Kilinc and C. Dehollain, "Intelligent Remote Powering," PCT/IB2013/056611, Aug. 13, 2013.

[16] E. G. Kilinc, C. Dehollain, and F. Maloberti, "Design and optimization of inductive power transmission for implantable sensor system," in Proc. 11th Int. Wokshop SM2ACD, Oct. 2010, pp. 1-5.

[17] E. G. Kilinc, F. Maloberti, and C. Dehollain, "Remotely powered telemetry system with dynamic power-adaptation for freely moving animals," in Proc. IEEE BioCAS, Nov. 2012, pp. 260-263.

[18] S. B. Lee, H.-M. Lee, M. Kiani, U.-M. Jow, and M. Ghovanloo, "An inductively-powered scalable 32-channel wireless neural recording system-on-a-chip for neuroscience applications," IEEE Trans. Biomed. Circuits Syst., vol. 4, no. 6, pp. 360-371, Dec. 2010.

[19] K. Finkenzeller, RFID Handbook. New York, NY, USA: Wiley, 2003.

[20] E. G. Kilinc, B. Canovas, F. Maloberti, and C. Dehollaini, "Intelligent cage for remotely powered freely moving animal telemetry systems," in Proc. IEEE ISCAS, May 2012, pp. 2207-2210.

[21] A. K. RamRakhyani and G. Lazzi, "Multicoil telemetry system for compensation of coil misalignment effects in implantable systems," IEEE Antennas Wireless Propag. Lett., vol. 11, no. 1, pp. 1675-1678, Jan. 2012.

[22] Altera, Corp. (2006). Arria II GX - FPGA Development Kit, San Jose, CA, USA [Online]. Available: http://www.altera.com

[23] Analog Devices, Inc. (2007). AD22151G - Linear Output Magnetic Field Sensor, Norwood, MA, USA [Online]. Available: http://www.analog.com

[24] Texas Instruments, Inc. (2013). ADC128S102 - 8-Channel, 500 ksps to 1 Msps, 12-bit A/D Converter, Dallas, TX, USA [Online]. Available: http://www.ti.com

[25] MISUMI Europa. (2013). RS106-C1-N - Single Axis Robot RS1, Taunus, Germany [Online]. Available: http://www.misumi-europe.com

[26] Microchip Technology, Inc. (1989). MCP23S18 - 16-Bit I/O Expander with Open-Drain Outputs, Chandler, AZ, USA [Online]. Available: http://www.microchip.com

[27] Fairchild Semiconductor, Corp. (2013). FODM8801C - High Temperature Phototransistor Optocoupler, San Jose, CA, USA [Online]. Available: http://www.fairchildsemi.com

[28] Webcraft, GmbH. (2006). S-05-02-N - Disc Magnet Diameter $5 \mathrm{~mm}$, Height $2 \mathrm{~mm}$, The Switzerland [Online]. Available: http://www.supermagnete.ch

[29] T. Givrad, J.-M. I. Maarek, W. H. Moore, and D. P. Holschneider "Powering an implantable minipump with a multi-layered printed circuit coil for drug infusion applications in rodents," Annal. Biomed. Eng., vol. 38, no. 3, pp. 707-713, Mar. 2010.

[30] S. Ghoreishizadeh, C. Baj-Rossi, S. Carrara, and G. De Micheli, "Nanosensor and circuit design for anti-cancer drug detection," in Proc. IEEE/NIH 5th LiSSA Workshop, Apr. 2011, pp. 28-33.

[31] Panasonic, Corp. (2008). EECENOF204 - 0.2 F, 3.3 V, Electric Double Layer Gold Capacitor, Osaka Prefecture, Japan [Online]. Available: http://www.panasonic.com

[32] A. M. Lauer, B. J. May, Z. J. Hao, and J. Watson, "Analysis of environmental sound levels in modern rodent housing rooms," Lab Animal, vol. 38, no. 5, pp. 154-160, May 2009. 
[33] H. Tamura, N. Ohgami, I. Yajima, M. Iida, K. Ohgami, N. Fujii, et al., "Chronic exposure to low frequency noise at moderate levels causes impaired balance in mice," PLOS ONE, vol. 7, no. 6, pp. e39807-1-e39807-3, Jun. 2012.

[34] N. Chaimanonart and D. J. Young, "A wireless batteryless in vivo EKG and body temperature sensing microsystem with $60 \mathrm{~Hz}$ suppression technique for untethered genetically engineered mice realtime monitoring," in Proc. IEEE EMBC Annu. Int. Conf., Sep. 2009, pp. 4872-4875.

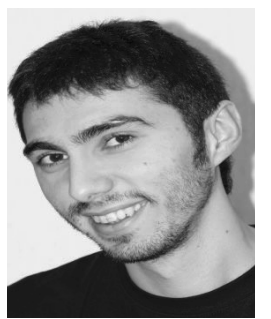

Enver Gurhan Kilinc received the B.Sc. degree in electronic engineering from Istanbul Technical University (ITU), Turkey, in 2008 and the M.Sc. degree in electrical and electronic engineering from Ecole Polytechnique Fédérale de Lausanne (EPFL), Switzerland, in 2010. In March 2010, he joined the Electronics Laboratory at EPFL as a Research Assistant in the field of RFIC design. He is currently working toward the Ph.D. degree in wireless power transmission and radio frequency identification (RFID) at the RFIC Group, Swiss Federale Institute of Technology (EPFL), Lausanne, Switzerland. His research interests include RF CMOS circuit design for wireless sensor systems and biomedical applications.

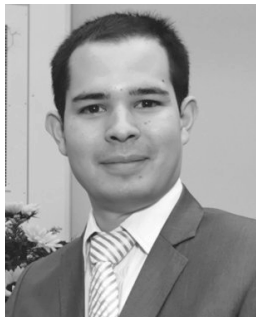

Gilbert Conus received the B.Sc. degree in electrical engineering from the Ecole Polytechnique Fédérale de Lausanne (EPFL), Lausanne, Switzerland, in 2010 and the M.Sc. degree in electronics and microelectronics engineering from the Ecole Polytechnique Fédérale de Lausanne (EPFL), Lausanne, Switzerland, in 2012. His research interests include digital, mixed-signal, and analog discrete electronics design as well as embedded and real-time embedded system design for universal domain applications.

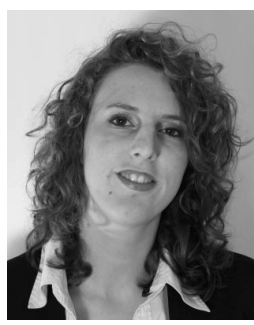

Chloé Weber received the B.Sc degree in electrical and electronic engineering from Ecole Polytechnique Fédérale de Lausanne (EPFL), Switzerland, in 2011. She is currently working towards the M.S. degree in the specialization electronics, micronanoelectronics at the EPFL. Her research interest includes real-time embedded system.

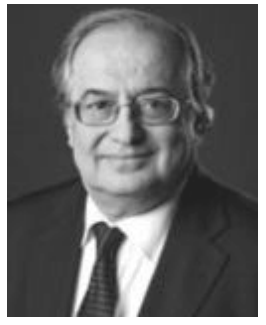

Basile Kawkabani received the M.S. degree in 1978 from SUPELEC, Ecole Supérieure d'Electricité, Paris, France, and the Ph.D. degree in 1984 in electrical engineering from the Swiss Federal Institute of Technology in Lausanne (EPFL). From 1992 to 2010, he was Lecturer and Research Associate at the EPFL Electrical Machinery Laboratory. $\mathrm{He}$ is currently a Senior Scientist with the STI Scientists Group (Electrical Machinery - EPFL), and Senior Member of the IEEE. His interests include modeling of power systems, power system stability and

control.

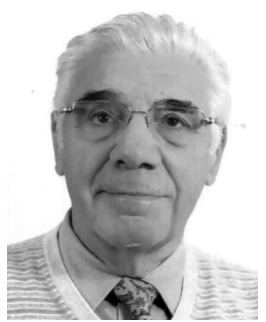

Franco Maloberti received the Laurea degree in physics (summa cum laude) from the University of Parma, Italy, in 1968 and the Dr. Honoris Causa degree in electronics from Inaoe, Puebla, Mexico in 1996. He was a Visiting Professor at ETH-PEL, Zurich in 1993 and at EPFL-LEG, Lausanne in 2004. $\mathrm{He}$ was a Professor of Microelectronics and Head of the Micro Integrated Systems Group, University of Pavia, Pavia, Italy, TI/J.Kilby Analog Engineering Chair Professor at the Texas A\&M University and the Distinguished Microelectronic Chair Professor at University of Texas at Dallas. Currently he is a Professor at the University of Pavia, Italy, and Honorary Professor, University of Macau, China SAR. His professional expertise is in the design, analysis and characterization of integrated circuits and analogue digital applications, mainly in the areas of switched capacitor circuits, data converters, interfaces for telecommunication and sensor systems, and portable power management. He has written more than 475 published papers, five books and holds 32 patents. He has been responsible for many research programs including ten ESPRIT projects and served the European Commission in many European Initiatives. He served the Academy of Finland on the assessment of electronic research. He served the National Research Council of Portugal for the research activity assessment of Portuguese Universities. He was a Member of the Advisory Board of INESCLisbon, Portugal. He is the Chairman of the Academic Committee of the Microelectronics Key Laboratory Macau, China.

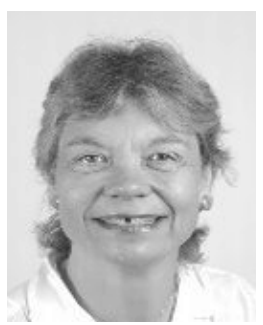

Catherine Dehollain received the Degree in electrical engineering in 1982 and the Ph.D. degree in 1995 from the Ecole Polytechnique Fédérale de Lausanne (EPFL), Lausanne, Switzerland. From 1982 to 1984, she was a Research Assistant at the Electronics Laboratories (LEG), EPFL. In 1984, she joined the Motorola European Center for Research and Development, Geneva, Switzerland, where she designed integrated circuits applied to telecommunications. In 1990, she joined EPFL as a Senior Assistant at the "Chaire des Circuits et Systémes," where she was involved in impedance broadband matching. Since 1995, she has been responsible for the RFIC Group, EPFL, for RF activities. She has been the Technical Project Manager of the European projects, CTI projects, and the Swiss National Science Foundation projects dedicated to RF wireless micropower sensor networks and mobile phones. Since 1998, she has been a Lecturer at EPFL in the area of RF circuits, electric filters, and CAD tools. Since 2006, she has been a "Maítre d'Enseignement et de Recherche," EPFL. She is an author or coauthor of four scientific books and 120 scientific publications. Her current research interests include low power analog circuits, biomedical remotely powered sensors, and electric filters. 